\title{
Late Presentation of Uterine Rupture Following Vaginal Birth After Cesarean Delivery: A Case Report
}

\author{
Lindsey A. Choi, DO ${ }^{1}$ Ariel A. Chung, MD, PharmD ${ }^{2}$ Brian Pierce, MD ${ }^{10}$ \\ ${ }^{1}$ Department of Obstetrics and Gynecology, Division of Maternal- \\ Fetal Medicine, Tripler Army Medical Center, Honolulu, Hawaii \\ 2 Department of Family Medicine, Tripler Army Medical Center, \\ Honolulu, Hawaii \\ Address for correspondence Brian Pierce, MD, Department of \\ Obstetrics and Gynecology, Tripler Army Medical Center, 1 Jarrett \\ White Road, Honolulu, HI 96859-5000 \\ (e-mail: brian.t.pierce.civ@mail.mil).
}

Am J Perinatol Rep 2020;10:e300-e303.

\author{
Abstract \\ Keywords \\ - uterine rupture \\ - trial of labor after \\ cesarean \\ - vaginal birth after \\ cesarean \\ - obstetrical \\ emergency \\ - uterine dehiscence
}

Background A trial of labor after cesarean delivery is associated with uterine rupture rates of 0.5 to $0.9 \%$, which can have devastating neonatal and maternal consequences. While uterine rupture typically occurs during labor, it can clinically manifest after delivery.

Case A 23-year-old multiparous female presented in labor at term. Her obstetrical history was significant for a prior low transverse cesarean delivery. She had an uncomplicated labor course and spontaneous vaginal delivery.

Immediately after delivery, she complained of severe right shoulder and left lower quadrant pain. Bedside ultrasound revealed a 10-cm, complex, adnexal mass adjacent to the uterus without free fluid. She was hemodynamically stable and appeared clinically well. On repeat ultrasound, the mass was unchanged; however, the patient now had free intraperitoneal fluid along the liver edge.

Emergent laparotomy revealed a uterine rupture along her prior hysterotomy with extension into the right uterine artery. A $10-\mathrm{cm}$ broad ligament hematoma ruptured posteriorly resulting in a 1-L hemoperitoneum. She received multiple blood products intraoperatively and recovered well postpartum.

Conclusion Delivery after trial of labor after cesarean delivery usually decreases acuity; however, these patients remain at risk for significant complications. Clinicians should continue to assess patients in the immediate postpartum period and proceed with surgical intervention if necessary.
Trial of labor after cesarean delivery (TOLAC) is an opportunity for women with a previous cesarean section to attempt a vaginal delivery. Currently in the United States, approximately $20 \%$ of women desire a TOLAC and $70 \%$ of those women are successful. ${ }^{1}$ The vaginal birth after cesarean delivery (VBAC) rate in the United States has been continuing to rise. ${ }^{2}$

VBAC is associated with several benefits including less invasive procedures, shorter duration of hospitalization, and decreased maternal morbidity. ${ }^{1,3}$ In addition, women who are empowered to pursue their desired mode of delivery

received

April 13, 2020

accepted

May 16, 2020
DOI https://doi.org/

10.1055/s-0040-1715175. ISSN 2157-6998. through TOLAC, have increased overall satisfaction. ${ }^{4}$ While VBAC provides many neonatal and maternal health benefits, TOLAC is also associated with significant risks and potentially devastating outcomes including uterine rupture, hemorrhage requiring blood transfusions, surgical exploration with hysterectomy, and rarely maternal and fetal death. ${ }^{1,3}$ Comprehensive patient counseling should be performed in all patients desiring TOLAC.

Uterine rupture occurs in approximately 0.5 to $0.9 \%$ of women attempting TOLAC with some studies reporting rates as high as $3.7 \%$ in women with more than one prior cesarean
Copyright $\odot 2020$ by Thieme Medical Publishers, Inc., 333 Seventh Avenue, New York, NY 10001, USA. Tel: +1(212) 760-0888.
License terms

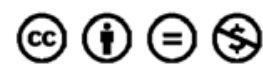


delivery. ${ }^{3,5}$ Clinical signs include vaginal bleeding, abdominal pain, loss of fetal station, palpable fetal parts in the abdomen, and changes in the fetal heart tracing, such as variable decelerations and fetal bradycardia. ${ }^{6}$ The most common location for a uterine rupture is along the previous uterine scar; however, uterine ruptures have also been reported in an unscarred uterus and can involve various anatomic locations. ${ }^{7-9}$ The most common timing of uterine rupture is intrapartum. ${ }^{6}$ Rarely, uterine rupture can be seen postpartum. ${ }^{10,11}$

Uterine rupture is an obstetrical emergency given the associated neonatal and maternal morbidity and mortality. ${ }^{6}$ Urgent delivery is required if uterine rupture is suspected during the antepartum or intrapartum period, and surgical management is necessary to address the uterine defect. If uterine rupture is diagnosed in the postpartum period, continued assessment is essential. Conservative management can be considered in a stable patient, but once a patient becomes unstable, surgical intervention is required.

\section{Case}

A 23-year-old gravida 2, para 1002 was admitted for oxytocin augmentation of latent labor at 40 weeks and 2 days of gestation in the setting of a previous uterine scar. Her surgical history was notable for a primary low transverse cesarean delivery for a nonvertex presenting twin. The type of uterine incision was confirmed by review of her surgical operative documentation. Her medical history was complicated by obesity (pre-pregnancy body mass index of $41 \mathrm{~kg}$ / $\mathrm{m}^{2}$ ), a history of postpartum hemorrhage requiring multiple blood product transfusions, and a history of preeclampsia in her prior pregnancy.

On admission, her cervix was dilated to $3 \mathrm{~cm}$ and she received an epidural for pain control. With subsequent oxytocin augmentation and artificial rupture of membranes, she progressed to complete dilation and effacement. After 1 hour in the second stage of labor, the patient had a successful VBAC. Immediately afterwards, she complained of new, sudden onset right shoulder, and left lower quadrant pain. She simultaneously had an increase in vaginal bleeding with estimated blood loss of $800 \mathrm{~mL}$, which responded to multiple uterotonics.

She was normotensive and nontachycardic with a heart rate in the 90s, and her abdominal exam revealed generalized and mild tenderness without distention. Due to her symptoms, she underwent a limited bedside abdominal sonogram by a maternal-fetal medicine specialist immediately after delivery. This revealed a complex appearing, $10-\mathrm{cm}$, right adnexal mass. No free fluid was noted in the pelvis or by the liver. A type and cross, complete blood count and coagulation studies were drawn; results were within normal limits. She was closely monitored for signs of intra-abdominal bleeding, as there was high clinical concern for uterine rupture given her history and pain.

Ongoing postpartum assessments were performed, and she remained normotensive and nontachycardic. Her abdomen remained soft and nondistended, and she reported an improvement in her abdominal pain. A total of 30 minutes after the initial bedside ultrasound, the same physician

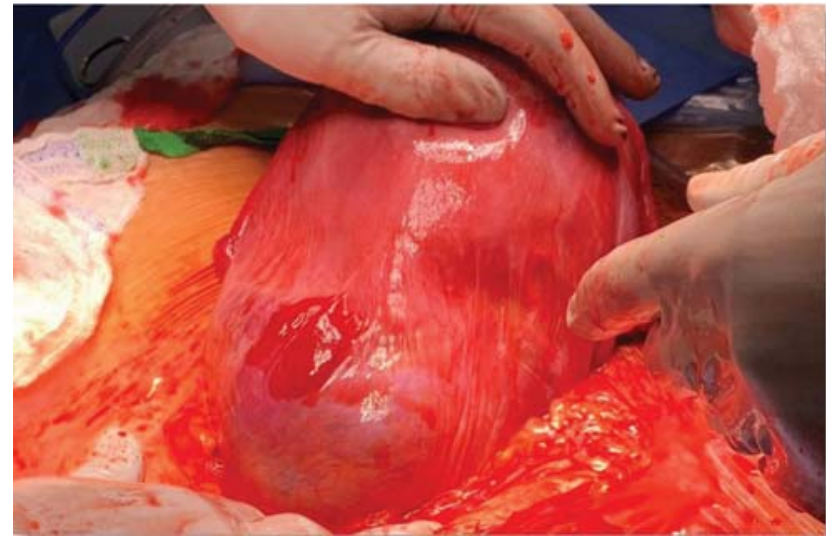

Fig. 1 A 10-cm broad ligament hematoma seen upon entry into the abdomen with exteriorization of the uterus.

repeated the evaluation. The previously noted right adnexal mass was stable in size and morphology; however, there was a new finding of free fluid in the right upper quadrant along the liver edge, measuring $4 \mathrm{~cm}$ in an anterior-posterior plane. With the new findings, she was counseled and consented for an emergent exploratory laparotomy given suspicion for hemoperitoneum.

An incision was made along her previous Pfannenstiel incision. Upon entry, the hemoperitoneum was confirmed and 1-L of blood was removed from the patient's abdomen. On further exploration, a large $10-\mathrm{cm}$ hematoma was seen in her right broad ligament (-Figs. 1 and $\mathbf{2}$ ) that was consistent with the complex mass seen on ultrasound. Two small $1 \mathrm{~cm}$ defects were noted in the posterior broad ligament, representing a broad ligament rupture, and resulting in the hemoperitoneum ( - Fig. 3). Incision into the anterior uterine serosa confirmed a dehiscence of the right half of the previous uterine incision. This dehiscence extended inferolateral for $10 \mathrm{~cm}$ into the right lateral margin of the lower uterine segment, cervix, and the right uterine artery.

The hematoma was evacuated, and the hysterotomy and inferior extensions were repaired with running, locking 0 -vicryl suture. Hemostasis of the right uterine artery laceration was achieved with proximal and distal ligation. Running,

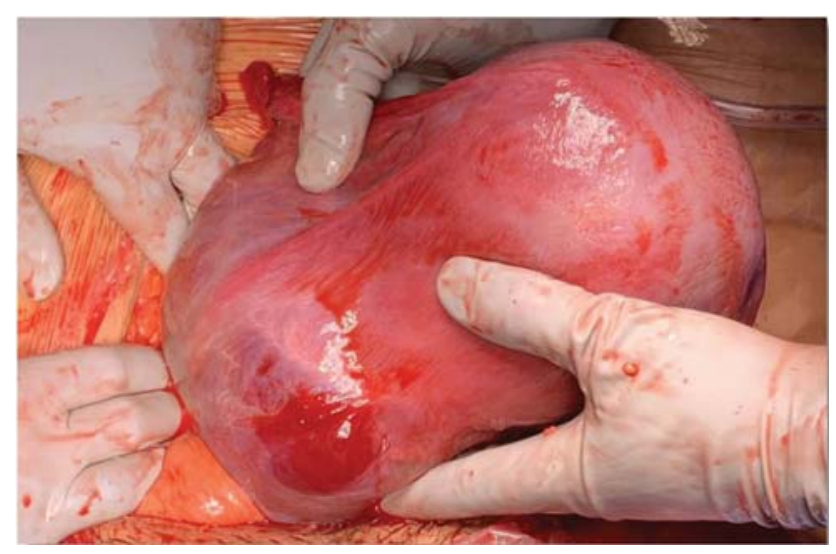

Fig. 2 Lateral view of the same $10-\mathrm{cm}$ broad ligament hematoma extending from anterior to posterior uterus. 


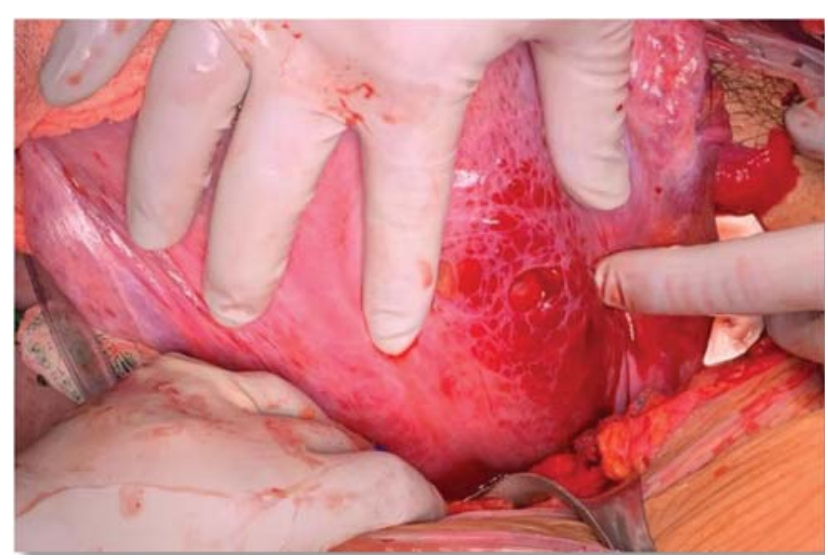

Fig. 3 Posterior view of the uterus with two small, posterior uterine serosal defects resulting in the 1-L hemoperitoneum.

locking 3-0 Vicryl sutures were also placed on the posterior broad ligament, incorporating the serosa defects. A hemostatic agent of microporous polysaccharide spheres (Arista) were applied along all sutures sites with excellent hemostasis obtained.

At this time, the uterus was atonic despite bimanual massage and she received additional uterotonics with improvement in her uterine tone. She received vasopressin for pressor support, and was transfused 4 units of packed red blood cells and 2 units of fresh frozen plasma intraoperatively. Once she was deemed stable, the uterus was returned to the abdomen and the fascia and the remainder of the abdomen were closed in the usual fashion. Due to the close proximity of the repair to the right ureter, a cystoscopy was performed at the end of the procedure, confirming bilateral ureteral patency.

Her estimated operative blood loss was $1,200 \mathrm{~mL}$ with a total delivery blood loss of $2,000 \mathrm{~mL}$. She received 24 hours of ampicillin/sulbactam postoperatively, and the remainder of her postpartum and postoperative course were uncomplicated. She was discharged home in good condition on postoperative day 3 .

\section{Discussion}

Typically, uterine rupture occurs during the course of labor. ${ }^{6}$ However, the diagnosis of uterine rupture can be delayed, occurring shortly after labor or several days after delivery. ${ }^{10,11}$ As uterine rupture can have devastating maternal and neonatal consequences, a high index of suspicion is essential in the postpartum period if a patient presents with signs and symptoms consistent with rupture.

Uterine ruptures have been described as either complete or incomplete in the literature. Complete uterine rupture involves a full thickness disruption of the uterine wall. In contrast, an incomplete uterine rupture (or uterine dehiscence) involves only the myometrium while preserving the serosa. This can result in decreased maternal and neonatal morbidity due to the confined nature of the defect. ${ }^{7}$ Of all the uterine ruptures that occur during labor, diagnosis is delayed in one fifth of cases. ${ }^{11}$ These cases have unique features including lower rates of prior uterine incisions, higher rates of epidural use, higher parity, and higher operative vaginal delivery rates. ${ }^{11}$ Rottenstreich et al performed a 13 -year retrospective study at a large tertiary academic center where 143 patients $(0.08 \%)$ were diagnosed with uterine rupture. Of all the cases, 29 (20.3\%) of the patients had a delayed diagnosis with a median time from delivery to operation of 4.5 hours. Maternal outcomes for the delayed diagnosis were significantly higher for blood transfusion rates, puerperal fever, and hysterectomy $(p>0.001)$. No differences were seen in neonatal outcomes, as the neonates were already delivered. ${ }^{11}$

In our patient, we suspect that the uterine rupture occurred immediately postpartum due to the absence of intrapartum fetal heart tracing abnormalities or maternal symptoms during the labor course. To the best of our knowledge, we present the first case of a suspected postpartum diagnosis of an incomplete anterior uterine rupture with progression into a complete posterior rupture through the broad ligament. Our case is unique in that the anterior incomplete rupture initially resulted in a large and stable broad ligament hematoma. We hypothesize that the uterine artery laceration continued to bleed into the broad ligament hematoma, ultimately progressing into a complete uterine rupture through the posterior broad ligament serosal defects. As demonstrated in our case, while the risk of uterine rupture may decrease following delivery, it can clinically manifest for the first time in the postpartum period. A clinician should have a high index of suspicion for uterine rupture if a patient experiences acute clinical changes such as severe abdominal pain, hemorrhage, tachycardia, and hypotension. In these patients, close observation is imperative, and immediate surgical intervention is necessary in an unstable patient.

Delivery of the neonate after TOLAC usually decreases physician acuity for uterine rupture. However, these patients still remain at risk for significant complications if diagnosis is delayed. Clinicians should continue to assess patients in the immediate postpartum period and proceed with surgical intervention if deemed necessary. This may prevent a delay in diagnosis and facilitate appropriate treatment and management with improved maternal outcomes.

Note

The views expressed in this abstract/manuscript are those of the authors and do not reflect the official policy or position of the Department of the Army, Department of Defense, or the U.S. Government.

Conflict of Interest

None declared.

\section{References}

1 Curtin SC, Gregory KD, Korst LM, Uddin SF. Maternal morbidity for vaginal and cesarean deliveries, according to previous cesarean history: new data from the birth certificate, 2013. Natl Vital Stat Rep 2015;64(04):1-13

2 Osterman MJK. Recent trends in vaginal birth after cesarean delivery: United States, 2016-2018. NCHS Data Brief, no 359. Hyattsville, MD: National Center for Health Statistics. Available at: https://www.cdc. gov/nchs/products/databriefs/db359.htm. Accessed March 5, 2020 
3 Landon MB, HauthJC, Leveno KJ, et al; National Institute of Child Health and Human Development Maternal-Fetal Medicine Units Network. Maternal and perinatal outcomes associated with a trial of labor after prior cesarean delivery. N Engl J Med 2004;351(25):2581-2589

4 Cheng YW, Eden KB, Marshall N, Pereira L, Caughey AB, Guise JM. Delivery after prior cesarean: maternal morbidity and mortality. Clin Perinatol 2011;38(02):297-309

5 Caughey AB, Shipp TD, Repke JT, Zelop CM, Cohen A, Lieberman E. Rate of uterine rupture during a trial of labor in women with one or two prior cesarean deliveries. Am J Obstet Gynecol 1999;181 (04):872-876

6 Leung AS, Leung EK, Paul RH. Uterine rupture after previous cesarean delivery: maternal and fetal consequences. Am J Obstet Gynecol 1993;169(04):945-950
7 Ahmadi F, Siahbazi S, Akhbari F. Incomplete cesarean scar rupture. J Reprod Infertil 2013;14(01):43-45

8 Sweeten KM, Graves WK, Athanassiou A. Spontaneous rupture of the unscarred uterus. Am J Obstet Gynecol 1995;172(06):1851-1855, discussion 1855-1856

9 Ofir K, Sheiner E, Levy A, Katz M, Mazor M. Uterine rupture: differences between a scarred and an unscarred uterus. Am J Obstet Gynecol 2004;191(02):425-429

10 Mavromatidis G, Karavas G, Margioula-Siarkou C, et al. Spontaneous postpartum rupture of an intact uterus: a case report. J Clin Med Res 2015;7(01):56-58

11 Rottenstreich M, Rotem R, Hirsch A, et al. Delayed diagnosis of intrapartum uterine rupture - maternal and neonatal consequences. J Matern Fetal Neonatal Med 2019;15:1-6 